\title{
Eutectic Anomaly of Compound Forming Bi-Te Liquids Studied by Electrical Resistivity Measurements
}

\author{
Yusuke Nakagawa*1 and Toshio Itami*2 \\ Division of Chemistry, Graduate School of Science, Hokkaido University, Sapporo 060-0810, Japan
}

\begin{abstract}
The electrical resistivity, $\rho$, of compound forming $\mathrm{Bi}-\mathrm{Te}$ system in the liquid state was studied by the dc-four probe method. Anomalous behavior of $\rho$ around the intermetallic compound $\mathrm{Bi}_{2} \mathrm{Te}_{3}$ in the previous study was not found. Anomalous behaviors of the temperature dependence of $\rho, d \rho / d T$, were found both at two eutectic compositions in this system. These behaviors were discussed based on the effective medium theory of electrical conduction. The concentration fluctuation at the eutectic composition ( 90 at\% Te) grows in the homogeneous liquid phase with the approach to the eutectic temperature.
\end{abstract}

(Received April 26, 2005; Accepted July 27, 2005; Published August 15, 2005)

Keywords: liquid metals, eutectic alloys, electrical resistivity, concentratyion fluctuations

\section{Introduction}

The Bi-Te system is the most important among thermoelectric materials because the intermetallic compound $\mathrm{Bi}_{2} \mathrm{Te}_{3}$ shows the highest figure of merit. The study of physicochemical properties of liquid $\mathrm{Bi}-\mathrm{Te}$ system closely relates to the single crystal growth process. Some anomalous behaviors of the electrical conductivity and viscosity ${ }^{1)}$ on their isotherms have been reported around the stoichiometric composition of intermetallic compound, $\mathrm{Bi}_{2} \mathrm{Te}_{3}$ (see Fig. 1, which is drawn by removing the ambiguous lines in the literature $\left.{ }^{2)}\right)$. In this phase diagram, ${ }^{2)}$ the existence of the two eutectic points is also noted. Recently one of authors found the growth of concentration fluctuations around the eutectic composition in the homogeneous liquid phase of $\mathrm{Ga}-\mathrm{Sb}, 3,4)$ In-Sb, ${ }^{4)} \mathrm{Ag}-\mathrm{Ge}$ and $\mathrm{Au}-\mathrm{Ge},{ }^{5)} \mathrm{Ag}-\mathrm{Si}$ and $\mathrm{Au}-\mathrm{Si}^{6-8)}$ systems. Okubo and Hirotsu ${ }^{9)}$ also found similar concentration fluctuations in the rapidly quenched glass state of eutectic $\mathrm{Pd}-\mathrm{Si}$ system. Therefore, it is interesting to clarify the characteristic features of homogeneous liquid phase for $\mathrm{Bi}-$ Te system by performing the accurate measurements of the electrical resistivity, $\rho$.

Generally it is not easy to obtain the exact experimental value of high temperature melts such as liquid $\mathrm{Bi}-\mathrm{Te}$ because of high temperature, lack of good container, etc. The measurement of the electrical resisitvity, $\rho$, can be performed accurately by the dc-four probe method if good containers and electrodes are present. The experimental chance for $\rho$ measurements in the laboratory is fundamentally abundant. The reliability of experimental data of $\rho$ can be kept to be very high by performing $\rho$ measurements as a detailed function of composition. In addition, the essential feature of the electrical resistivity is the scattering phenomena of conduction electron wave by the constituent ions. This wave scattering phenomena is quite similar to the principle of the conventional structure analysis of liquids based on the scattered wave phenomena of synchrotron X-ray or neutrons by the constituent atoms. At present, these conventional methods of liquid structure analysis do not always possess the

\footnotetext{
${ }^{* 1}$ Graduate Student, Hokkaido University

${ }^{* 2}$ Corresponding Author, E-mail: itami@sci.hokudai.ac.jp
}

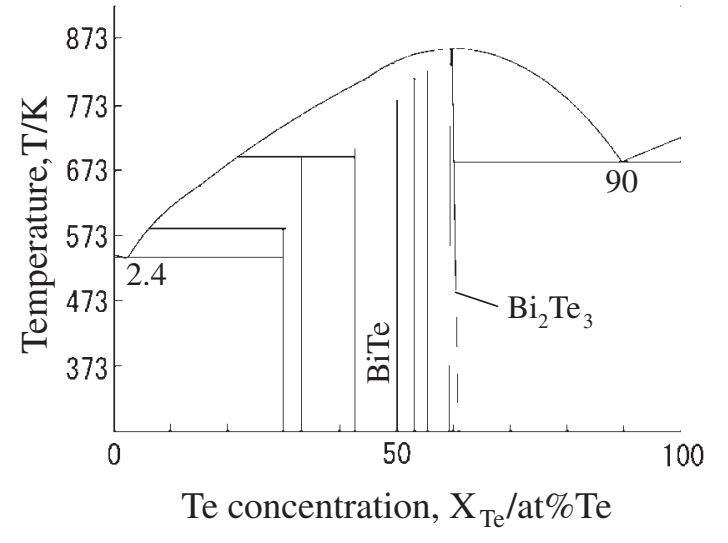

Fig. 1 Phase diagram of $\mathrm{Bi}-\mathrm{Te}$ system. ${ }^{2)}$

sufficient accuracy. Moreover, experimental chances of such structure analysis are not always abundant because of the use of big experimental facility. We can expect that measurements of $\rho$ as a detailed function of composition are effective to observe the evidentiary base of the structure change of liquids.

In this paper, the evidentiary base of concentration fluctuations in the homogeneous liquid of eutectic system, $\mathrm{Bi}-\mathrm{Te}$, was investigated by $\rho$ measurements. ${ }^{10)}$ The concentration fluctuation was analyzed based on the effective medium theory.

\section{Experimental}

The electrical resistivity, $\rho$, was measured by the dc-four probe method. The specially designed cell ${ }^{3,11,12)}$ was employed. The important point in the present study is the accurate measurement of sample temperature. This was realized by immersing the thermocouples into liquid samples in terms of small glass tubes. Tungsten wire electrodes were sealed into Pyrex glass cell by the fusion sealing technique. The purity of metal used was $99.99 \%$ for Bi and $99.9999 \%$ for Te respectively. The weighed amount of $\mathrm{Bi}$ and $\mathrm{Te}$ was inserted into Pyrex glass cell, which was sealed under vacuum. The measurements of $\rho$ were performed on cooling 


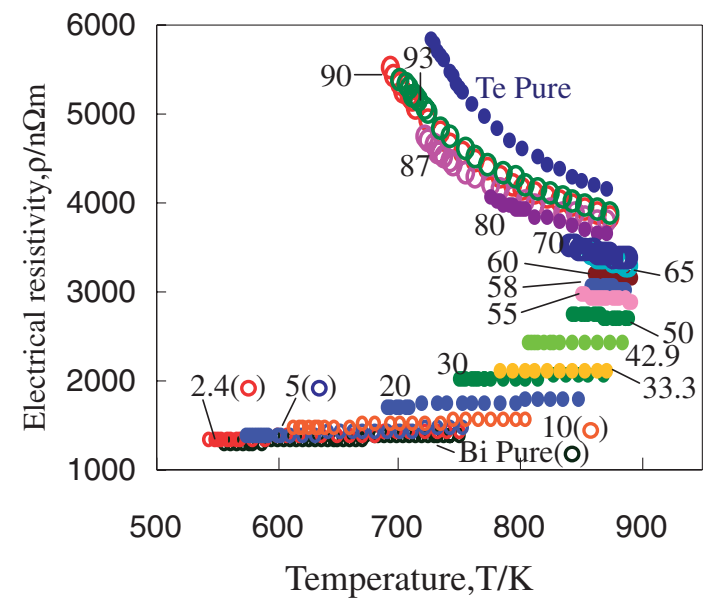

Fig. 2 The temperature dependence of the electrical resistivity, $\rho$, of liquid $\mathrm{Bi}-\mathrm{Te}$ alloys (The number attached to the line is Te concentration (at\% Te)).

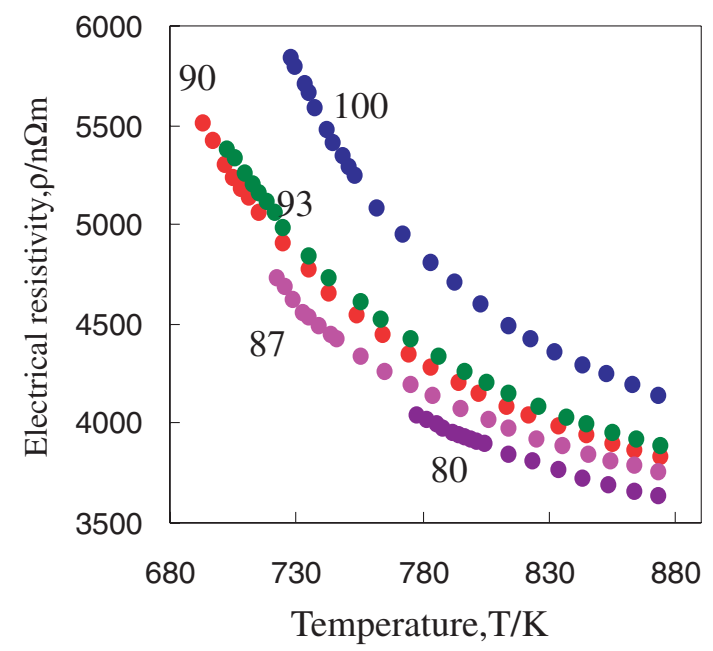

Fig. 3 Close up around the eutectic composition (90 at\% Te) of Fig. 2 (The number attached to the line is Te concentration (at\% Te)).

process. The experimental data of $\rho$ was expressed as the fourth order function of $T$ by the least square method except for 50 at $\% \mathrm{Te}-70$ at $\% \mathrm{Te}$, for which the second order function of $T$ was adopted because of the lack of wide temperature range. The $\rho$ and its temperature coefficient, $d \rho / d T$, were determined by this experimental formula.

\section{Results}

Figure 2 shows the measured temperature dependence of $\rho$. The magnified view of the measured $\rho$ around 90 at $\%$ Te $\left(X_{\text {eut }}^{\mathrm{I}}\right.$ : eutectic composition I) was shown in Fig. 3 . Figure 4 depicts the concentration dependence of $\rho$. Around $\mathrm{Bi}_{2} \mathrm{Te}_{3}$ composition, the $\rho$ does not show the double maximum reported by Glazov et al. ${ }^{1)}$ The present data shows a very slight maximum around the $\mathrm{Bi}_{2} \mathrm{Te}_{3}$ and distinctly a small maximum at $X_{\text {eut }}{ }^{\mathrm{I}}$, particularly along the liquidus line. This small maximum at the $X_{\text {eut }}^{\mathrm{I}}$ is certain judging from the reproducibility of $\rho$ measurements, $0.2 \mu \Omega \mathrm{cm}$ (Note the relation $10 \mathrm{n} \Omega \mathrm{m}=1 \mu \Omega \mathrm{cm})$. The concentration dependence

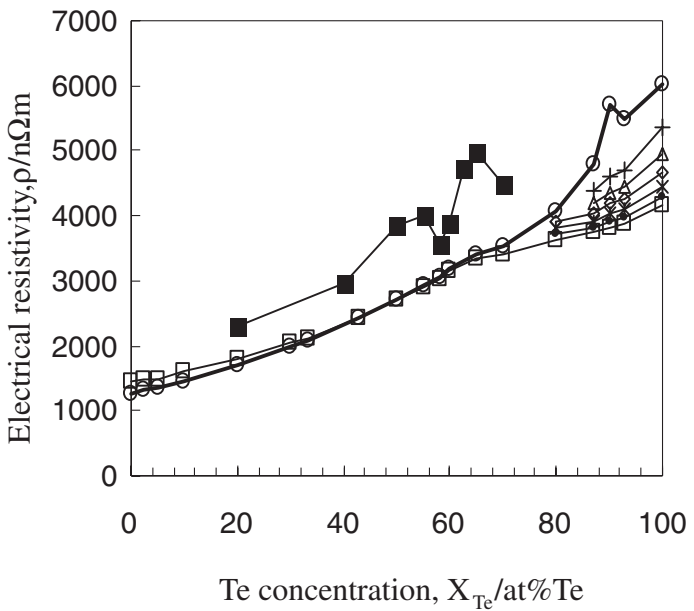

Fig. 4 Concentration (at $\% \mathrm{Te}$ ) dependence of the electrical resistivity, $\rho$, of liquid Bi-Te alloys ( $\square: 873 \mathrm{~K} ; \bullet: 848 \mathrm{~K} ; \times: 823 \mathrm{~K} ; \diamond: 798 \mathrm{~K} ; \triangle: 773 \mathrm{~K}$; +: $748 \mathrm{~K}$; $\bigcirc$ : liquidus; $\mathbf{\square}$ : Glazov et al. ${ }^{1)}$ at $\left.873 \mathrm{~K}\right)$.

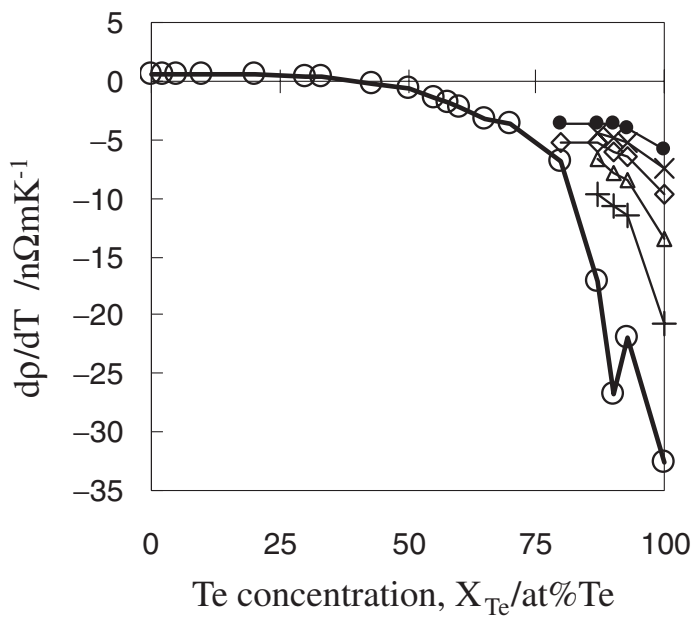

Fig. 5 Concentration (at $\% \mathrm{Te}$ ) dependence of the temperature coefficient, $d \rho / d T$, of liquid Bi-Te alloys $(\bigcirc 84 \mathrm{~K} ; \times: 823 \mathrm{~K} ; \diamond: 798 \mathrm{~K} ; \triangle: 773 \mathrm{~K}$; $+: 748 \mathrm{~K}$; $\bigcirc$ : liquidus).

of the temperature coefficient of $\rho, d \rho / d T$, is shown in Fig. 5. The $d \rho / d T$ shows a minimum at the $X_{\text {eut }}{ }^{\mathrm{I}}$. Figure 6 depicts the magnified view of the concentration dependence of $d \rho / d T$ around the eutectic composition, 2.4 at $\%$ Te $\left(X_{\text {eut }}{ }^{\mathrm{II}}\right.$ : eutectic composition II). The slight minimum of $d \rho / d T$ can be seen also at the $X_{\text {eut }}{ }^{\text {II }}$. These anomalies of $d \rho / d T$ may be certain judging from the experimental reproducibility, $0.24 \mu \Omega \mathrm{cmK}^{-1}$ for $X_{\text {eut }}{ }^{\mathrm{I}}$ and $5 \times 10^{-6} \mu \Omega \mathrm{cmK}^{-1}$ for $X_{\text {eut }}$ II . A slight minimum of $d \rho / d T$ can be seen around the composition of $\mathrm{Bi}_{2} \mathrm{Te}_{3}$.

\section{Discussions}

The anomaly of the $\rho$ around the composition of intermetallic compound, $\mathrm{Bi}_{2} \mathrm{Te}_{3}$, reported by Galzov et al. ${ }^{1)}$ could not be found. Only a very slight maximum (or hump) could be found around this composition. This difference may be derived from the difference of the adopted method for $\rho$ measurements. Glazov et al. ${ }^{1)}$ adopted the electrodeless 


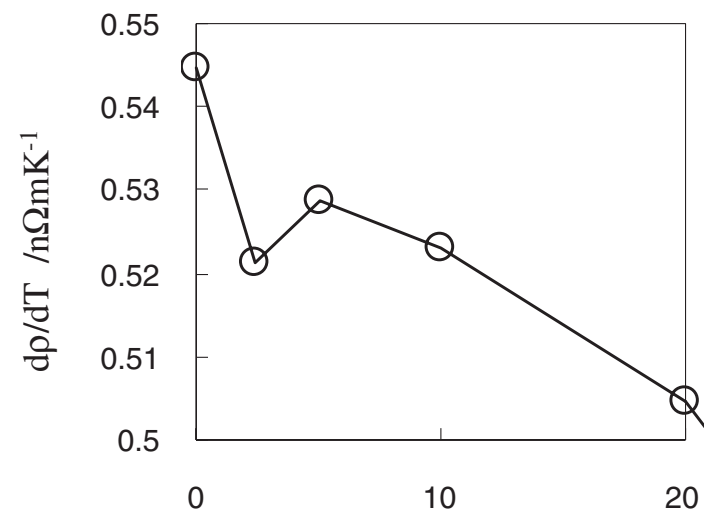

Te concentration, $\mathrm{X}_{\mathrm{Te}} / \mathrm{at} \% \mathrm{Te}$

Fig. 6 Close up around the eutectic composition (2.4 at\%Te) of Fig. 5.

method, which is an indirect method based on the rotating magnetic field. In the present study, the dc 4-probe method, which is the direct method for $\rho$ measurements, was employed. The particular feature found in this study for liquid Bi-Te system was the anomaly of the $d \rho / d T$ around both two eutectic compositions, $X_{\text {eut }}{ }^{I}$ and $X_{\text {eut }}{ }^{\text {II }}$. This reminds us the recent finding for some eutectic systems, namely the growth of the large concentration fluctuations in the homogeneous liquids near the eutectic composition. This conclusion was obtained by the experimental fact that the negative $d \rho / d T$ appears only near the eutectic composition on cooling process of liquid $\mathrm{Ga}-\mathrm{Sb}$ system. ${ }^{3,4)}$

The negative $d \rho / d T$ is known to appear in the case of $\mathrm{Z}^{*} \cong 2$ ( $\mathrm{Z}^{*}$ : average valence). ${ }^{11,12)}$ However, for eutectic composition, 88.2 at\% Ga, in the $\mathrm{Ga}-\mathrm{Sb}$ system, the $\mathrm{Z}^{*}$ cannot be close to 2 . The anomaly around the eutectic composition of $\mathrm{Ga}-\mathrm{Sb}$ system is the particular feature closely related to the existence of the eutectic point. In addition, the negative $d \rho / d T$ for liquid $\mathrm{Ga}-\mathrm{Sb}$ system was successfully explained by the effective medium theory in which the existence of the concentration fluctuations, or solid like domains of intermetallic GaSb like and those of $\mathrm{Sb}$ like, was assumed in the homogeneous liquid phase. The Ga-Sb system is essentially metal-semiconductor eutectic system between intermetallic $\mathrm{GaSb}$ and $\mathrm{Sb}$. As similar metal-semiconductor systems, In$\mathrm{Sb}, \mathrm{Au}-\mathrm{Ge}, \mathrm{Au}-\mathrm{Si}, \mathrm{Ag}-\mathrm{Ge}$ and Ag-Si systems were studied by the $\rho$ measurements. ${ }^{4-8)} \mathrm{Au}-\mathrm{Ge}$ and $\mathrm{Au}-\mathrm{Si}$ systems are deep eutectic systems whose melting temperatures are far lower than those of respective constituent components. The anomaly and the negative $d \rho / d T$ were found in the homogeneous liquids near the eutectic composition for all these systems including deep eutectic systems. In addition, the evaluated degree of concentration fluctuations at the eutectic point for these systems was well summarized by a single tendency as the function of parameters relating to the temperature depth, $\Delta T$, of eutectic temperature measured from the melting temperature of constituent semiconductor phase and the composition difference, $\Delta \mathrm{X}$, between the eutectic composition and the composition of the constituent semiconductor phase. Therefore, the anomaly at the eutectic composition for the $\mathrm{Bi}-\mathrm{Te}$ system may be derived also from the growth of concentration fluctuations.
As shown in Figs. 3 and 4, the enhanced tendency of $\rho$ and the depression tendency of $d \rho / d T$ for 90 at $\%$ Te (the eutectic composition) is far distinct with the approach to the eutectic temperature compared with other compositions. It is straightforward to consider that this may be derived from the appearance of domains with large resistivity(as concentration fluctuations), namely solid like domains of Te like and $\mathrm{Bi}_{2} \mathrm{Te}_{3}$ like. Such a view point has been already applied successfully to $\mathrm{Ga}-\mathrm{Sb}$, In-Sb, $\mathrm{Au}-\mathrm{Si}, \mathrm{Au}-\mathrm{Ge}, \mathrm{Ag}-\mathrm{Si}$ and Ag-Ge systems. ${ }^{3-8)}$ These domains can be assumed as microscopic prefreezing phenomena in the homogeneous liquid phase for the eutectic solidification. It is very important to grasp the evidence of the existence of such domains by a small angle X-ray or neutron scattering experiments. However, at present, such experiments have not yet been performed. Here we note the recent experimental study of Okubo and Hirotsu, ${ }^{9)}$ which showed the existence of nanocrystal structure of primary solidification phase in the rapidly quenched glass of eutectic Pd-Si system. This may provide some evidence for the existence of concentration fluctuations which corresponds to the prefreezing phenomena of eutectic solidifications. In addition, there is a fact that the anomalous behavior of the $\rho$ of many metal-semiconductor eutectic systems $^{3-8)}$ in the liquid state was able to be explained by the concentration fluctuation analysis under the assumption of such solid like domains in the homogeneous liquid phase. It is quite natural to analyze the behavior of $\rho$ based on the fluctuation model for liquid $\mathrm{Bi}-\mathrm{Te}$ system, which has two eutectic points, one is at 2.4 at $\% \mathrm{Te}$ and the other is at 90 at $\%$ Te, At first, we discuss the anomaly around the 90 at\% Te, which is the eutectic composition between intermetallic $\mathrm{Bi}_{2} \mathrm{Te}_{3}$ and Te.

Here it is assumed that the Te like and $\mathrm{Bi}_{2} \mathrm{Te}_{3}$ like domains are assumed to exist in the homogeneous liquid phase. The resistivity of such inhomogeneous phase can be represented by the effective medium theory, which assumes that the average (mean) medium for the present inhomogeneous system is identical to the volume fraction average of Te like phase $\left(\rho_{1}, \phi_{1}\right), \mathrm{Bi}_{2} \mathrm{Te}_{3}$ like phase $\left(\rho_{2}, \phi_{2}\right)$, and homogenous phase $\left(\rho_{3}, \phi_{3}\right)$. The $\rho_{\mathrm{i}}$ and $\phi_{\mathrm{i}}$ indicate respectively the $\rho$ and the volume fraction of phase i. Explicit formula for the present three phases case is written as follows:

$$
\begin{gathered}
\phi_{1}\left(\frac{1 / \rho_{1}-1 / \rho_{m}}{1 / \rho_{1}+2 / \rho_{m}}\right)+\phi_{1}\left(\frac{1 / \rho_{2}-1 / \rho_{m}}{1 / \rho_{2}+2 / \rho_{m}}\right) \\
+\phi_{1}\left(\frac{1 / \rho_{3}-1 / \rho_{m}}{1 / \rho_{3}+2 / \rho_{m}}\right)=0 .
\end{gathered}
$$

This equation for three phases case was obtained by extending straightforwardly the expression for two phasaes case. ${ }^{15,16)}$ The $\rho_{\mathrm{i}}$ of semiconductor like domains was estimated at the liquidus temperature by the extrapolation of solid $\rho$ data. ${ }^{17,18)}$ The estimated values for $\rho_{1}$ and $\rho_{2}$ are respectively $0.2 \Omega \mathrm{cm}$ and $800 \mu \Omega \mathrm{cm}$. The relations, $\rho_{1} \gg$ $\rho_{m}, \rho_{2}>\rho_{m}$ and $\rho_{3}-\rho_{m}$ hold. ${ }^{17,18)}$ Under this condition, eq. (1) can be converted into eq. (2) for the estimation of the volume fraction of concentration fluctuations, $\phi_{1}+\phi_{2}$.

$$
\phi_{1}+\phi_{2}=\frac{1}{1-\delta}\left(\frac{2 \Delta \rho}{3 \rho_{m}}\right) \text {. }
$$


In these equations, $\Delta \rho=\rho_{m}-\rho_{3}$ and $\delta=-3 k / 2(k+2)$. The $k$ is defined as $k=\rho_{m} / \rho_{2}=\sigma_{2} / \sigma_{m}$. If we can know $\Delta \rho$ and $k$, we can estimate the volume fraction of concentration fluctuations, $\phi_{1}+\phi_{2}$.

In the previous studies, ${ }^{3-8)}$ the $\Delta \rho$, namely the deviation of $\rho$ from $\rho_{3}$ (the $\rho$ of homogeneous phase with negligible concentration fluctuations), can be easily estimated by the linear extrapolation of $\rho$ in the high temperature range to the low temperature range based on the fact that the $T$ dependence of $\rho$ is linear at the high temperature range where the concentration fluctuation is absent and the deviation of $\rho$ from the linear law increases on cooling process. However, the estimation of the $\Delta \rho$ for the present $\mathrm{Bi}-\mathrm{Te}$ system is difficult because the $\rho$ does not change linearly all over the temperature range studied here (see Fig. 1). We note in Fig. 3 that the temperature dependence of $\rho$ for $80 \mathrm{at} \% \mathrm{Te}$ is quite similar to that for 87 at $\%$ Te. Therefore, these $\rho-T$ relations (80 and 87 at $\% \mathrm{Te}$ ) may be free from the concentration fluctuations. The temperature variation of $\rho$ for 87 at $\%$ Te was adopted as the $\rho-T$ relation with no concentration fluctuations. In addition, the temperature dependence of 90 at $\%$ Te (eutectic composition) is large particularly with the approach to the eutectic temperature. We estimate the degree of concentration fluctuation only for the eutectic composition, 90 at $\%$ Te. The procedure of this estimation is described below.

1) The $\rho$ of 87 at $\%$ Te was extrapolated into lower temperature range (down to $693 \mathrm{~K}$ ) by using the experimental formula of fourth order function of temperature.

2) This extrapolation curve was put on the $\rho-T$ relation of 90 at $\%$ Te so that the $\rho$ at $873 \mathrm{~K}$ agrees with each other. This shifted extrapolated curve was assumed to be $\rho_{3}-T$ relation.

3) The deviation of $\rho_{m}$ from $\rho_{3}$, that is $\Delta \rho$, was estimated from the $\rho-T$ relation at 90 at $\% \mathrm{Te}\left(\rho_{m}-T\right.$ relation $)$ and $\rho_{3}-T$ relation described above.

4) The factor $k$ is assumed to be $0.5 .{ }^{17}$ )

5) By using eqs. (2)-(4), the degree of concentration fluctuation was evaluated.

Figure 7 indicates that the volume fraction of the concentration fluctuations on cooling process increases rapidly up to $5 \%$. However, this estimation is simply an order estimation under difficult conditions derived from many simplifications and approximations. What authors have to say is only the existence of concentration fluctuations in homogeneous liquid phase of eutectic systems. These concentration fluctuations are considered to be derived from the microscopic prefreezing in eutectic liquids. This was supported also by the observation that the supercooling of homogeneous liquid phase, which was detected during $\rho$ measurements on cooling process, is absent at both the eutectic compositions, 2.4 at\% Te and 90 at\% Te. Nothing to say, the structure of concentration fluctuations must be analyzed qualitatively, for example, by small angle scattering experiments. The present study is expected to provide a motivation for such studies. The anomaly of $d \rho / d T$ for 2.4 at $\%$ Te may be analyzed in a similar manner to that for 90 at $\%$ Te.

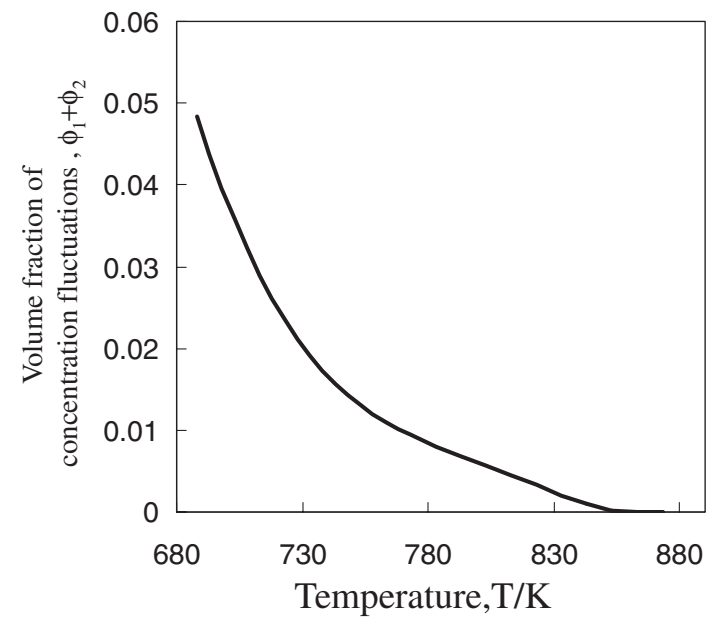

Fig. 7 The temperature dependence of the volume fraction $\left(\phi_{1}+\phi_{2}\right)$ of the concentration fluctuations at the eutectic composition (90 at $\% \mathrm{Te})\left(\phi_{1}\right.$ : volume fraction of $\mathrm{Bi}$ like domain; $\phi_{2}$ : volume fraction of $\mathrm{Bi}_{2} \mathrm{Te}_{3}$ like domain).

\section{Conclusions}

In liquid $\mathrm{Bi}-\mathrm{Te}$ system, the anomaly derived from the existence of the eutectic point is dominant compared with the effect of compound forming. This indicates the existence of concentration fluctuations in the homogeneous liquids of eutectic systems.

\section{REFERENCES}

1) V. M. Glazov, S. N. Chizhevskaya and N. N. Glagoleva: Liquid Semiconductors (Plenum Press, New york, 1969) pp. 294-300.

2) ASM International Binary Alloy Phase Diagrams, 2nd Ed., The Materials Information Society, Materials Park, OH (on CD-ROM).

3) H. Aoki, T. Shibata and T. Itami: J. Phys.: Condens. Matter 11 (1999) 10313-10322.

4) H. Aoki, K. Hotozuka and T. Itami: J. Non-Cryst. Solids 312-314 (2002) 222-226

5) M. Ikeda, T. Shibata, H. Aoki and T. Itami: J. Non-Cryst. Solids 312314 (2002) 217-221.

6) T. Itami, H. Aoki, T. Shibata, M. Ikeda and K. Hotozuka: submitted in J. Non-Cryst. Solids.

7) T. Itami, H. Aoki, T. Shibata, M. Ikeda and K. Hotozuka: Space Utilization Research 19 (2003) 216-218.

8) T. Itami, H. Aoki, T. Shibata, M. Ikeda and K. Hotozuka: Space Utilization Research 20 (2004), 250-252.

9) T. Ohkubo and Y. Hirotsu: Phys. Rev. 67B (2003) 094201-1-9.

10) Y. Nakagawa and T. Itami: Collected Abstracts of the 2005 Spring Meeting of the Japan Inst. Metals (2005) p. 272.

11) T. Masaki, T. Itami, K. Kuribayashi, M. Hinada and K. Kawasaki: Rev. Sci. Instrum. 67 (1996) 2325-2331.

12) T. Itami, N. Takahashi and M. Shimoji: J. Phys. F: Metal Phys. 13 (1983) 1225-1232.

13) G. Busch and H.-J. Guntherodt: Phys. Condens. Matter. 6 (1967), 325.

14) M. Shimoji: "Liquid Metals" (Academic Press, London, New York San Francisco 1977), p. 275-276.

15) R. Landauer: J. Appl. Phys. 23 (1952) 779.

16) N. E. Cusack: "The Physics of Structurally Disordered Matter: An Introduction" (AdamHilger, Bristol and Philadelphia, 1987) p. 235238.

17) L. I. Berger: "Semiconductor Materials" (CRC Press, Boca Raton New York London Tokyo, 1997), p. 363.

18) S. Tanaka: Oyo Butsuri 40 (1971) 595-615. 